\title{
DATENSCHUTZ
}

\section{Wir haben etwas zu verbergen!}

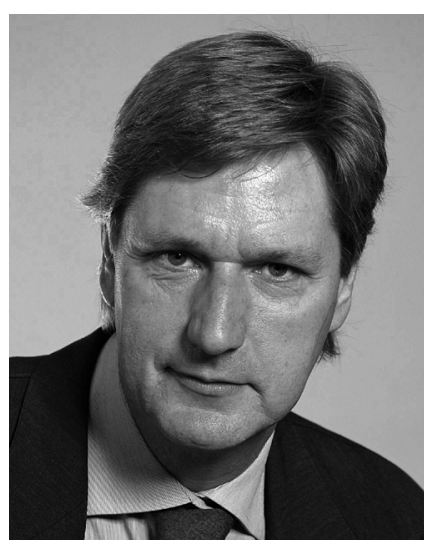

VON GERFRIED RIEKEWOLT Gerfried Riekewolt ist DiplomIngenieur (FH) und Diplom Sozialpädagoge (FH) mit Studienschwerpunkt soziale Organisation. Er arbeitete in der beruflichen Rehabilitation, war Regionalleiter eines Softwarehauses der Sozialbranche, Seniorconsultant einer bundesweiten Unternehmensberatung und ist seit $2003 \mathrm{im}$ Bereich Datenschutz und Sozialdatenschutz sowie im Projektmanagement selbständig tätig. Als Datenschutzbeauftragter und pers. Cert. Datenschutzauditor (TÜV) ist er Mitglied im Ausschuss Berufsbild des Berufsverbandes der Datenschutzbeauftragten Deutschland e. V. (BvD). www.riekewolt.de

\author{
Datenschutz wird selbstverständlich auch in der \\ Sozialwirtschaft befürwortet. Fragt man etwas in die Tiefe, \\ werden die Überprüfbarkeit und die Unsicherheiten bei \\ dem Thema spürbar. Das Selbstverständnis, Datenschutz \\ "aus dem Bauch heraus" zu praktizieren, ist für eine \\ unternehmerische Rechtssicherheit jedoch nicht ausreichend.
}

Es wundert nicht, dass Datenschutz so wenig "gelebt« wird: Das Datenschutzrecht ist für den juristischen Laien schwer verständlich. Dem Aufbau der Gesetze zum Datenschutz mit dem Grundansatz des Verbots zur Datenerhebung und Datenverarbeitung mit Erlaubnisvorbehalt ( $\$ 4$ Abs.1 BDSG), stehen die private Freizügigkeit der Selbstdarstellung und die praktische Kommunikation mit WhatsApp u. a. entgegen.

Dem »Ich habe ja nichts zu verbergen « steht in der Sozialen Arbeit ein "Wir haben etwas zu verbergen « gegenüber: Soziale Einrichtungen, Organisationen und Vereine haben im Interesse der Klientel einen sorgsamen Umgang mit allen personenbezogenen Daten sicher zu stellen. Und dies nicht erst seit der Affäre um »Prism«. Gründe finden sich im Selbstverständnis sozialer Organisationen, im Menschenbild, im Qualitätsanspruch und natürlich im Datenschutz im Interesse der Klientel.

Klientendaten enthalten immer eine besonderen Sensibilität: Eine persönliche Not- oder Ausnahmesituation, eine Beratung oder eine Inanspruchnahme von Maßnahmen sind Merkmale sozialer Dienstleistungen. Ganz gleich, ob diese den Gesundheitsdaten (vgl. \$3 Abs. 9 BDSG) zuzuordnen sind oder nicht, ein sorgfältiger Umgang mit diesen besonderen Arten personenbezogener Daten wird vom Klientel erwartet. So gesehen, ist die Einhaltung des Bundesdatenschutzgesetzes (BDSG) auch aus diesen Gründen der selbstverständliche Mindeststandard.

\section{Die "Verantwortliche Stelle»}

Soziale Einrichtungen als »soziale Leistungserbringer « sind den »nicht öffentlichen Stellen« zuzuordnen $(\mathbb{S} 2$ Abs.4 BDSG) und haben Datenschutz in Eigenverantwortung umzusetzen ( $\int 1$ Abs. 2 BDSG). Dies obliegt der Organisation der "Verantwortlichen Stelle $(\mathbb{S} 3$ Abs. 7 BDSG) in der Benennung der zuständigen Leitungsebene $(\mathbb{S} 4 \mathrm{e}$ Nr. $1 \mathrm{ff}$. BDSG). Damit stehen Geschäftsführung und Vorstand immer in der Verantwortung zur Sicherstellung des Datenschutzes.

Die häufig gestellte Frage, ob ein Datenschutzbeauftragter überhaupt $\mathrm{zu}$ bestellen ist, wird nicht nur mit den in $\mathbb{S} 4 \mathrm{f}$. BDSG zitierten Mindestzahlen von mehr als 20 oder 10 Personen, »die ständig mit der automatisierten Verarbeitung personenbezogener $\mathrm{Da}$ ten beschäftigt sind « beantwortet. Die Bestellpflicht eines Datenschutzbeauftragten ergibt sich auch aus der Pflicht der Vorabkontrolle (vgl. $\int 4$ d Abs. 5 BDSG):

»Eine Vorabkontrolle ist insbesondere durchzuführen, wenn

1. besondere Arten personenbezogener Daten verarbeitet werden oder

2. die Verarbeitung personenbezogener Daten dazu bestimmt ist, die Persönlichkeit des Betroffenen zu bewerten einschließlich seiner Fähigkeiten, seiner Leistung oder seines Verhaltens ..." 
Jede individuelle Hilfeplanung enthält genau diese Inhalte, ganz gleich in welcher sozialen Fachrichtung. Diese Prüfung der Einhaltung der Vorabkontrolle obliegt dem Datenschutzbeauftragten (\$ 4d Abs. 6 BDSG). Somit müssen alle sozialen Einrichtungen, unabhängig von der Größe oder der Zahl der Klienten, einen Datenschutzbeauftragten bestellen. Sollte es wirklich Gründe dafür geben, keinen Datenschutzbeauftragten bestellen zu müssen, »hat der Leiter der nicht öffentlichen Stelle« in jedem Fall den Datenschutz zumindest »in anderer Weise sicherzustellen « ( $\mathbb{S} 4 \mathrm{~g}$ Abs. 2 BDSG) und dies intern rechtssicher zu begründen und zu dokumentieren.

\section{Datenschutz und Sozialdatenschutz}

Die Umsetzung sozialer Dienstleistungen nach einem der zwölf Sozialgesetzbücher liegt zwar grundsätzlich in eigener Verantwortung und in der eigener Gestaltungsfreiheit sozialer Dienstleister. Die Vielfalt unterschiedlicher Angebote ist in der Sozialgesetzgebung ausdrücklich gewünscht. Dennoch fehlen in der Grundlage zum Sozialdatenschutz ( $\int 35$ SGB I) die sozialen Leistungserbringer. Der Sozialdatenschutz im Sozialgesetzbuch berührt zunächst nur die sozialen Leistungsträger. Die Übertragung des Sozialdatenschutzes auf soziale Dienstleister erfolgt erst über individuelle Vertragsvereinbarungen, Anlagen zu Verträgen u. a. verbindlichen Vorgaben.

\section{Compliance}

Das System Datenschutz in Unternehmen, auch in sozialen Einrichtungen, basiert auf einem System der Selbstkontrolle mit Aufsichtsbehörde: in Eigenverantwortung sind die Aufgaben zum Datenschutz zu erledigen und durch eine mit dem Qualitätsmanagement vergleichbaren Dokumentation der Umsetzung zu belegen (vgl. $\$ 4 \mathrm{~g}$ BDSG). Dazu gehören auch die Dokumentation, die Rechte der Betroffenen sicherstellen (\$S 6, 33-36 BDSG), die sogenannte technische und organisatorische Maßnahmen zum Datenschutz, einschließlich Verschlüsselungstechnologien, betreffen ( $\mathbb{S} 9$ BDSG und Anlage), die Datenverarbeitung im Auftrag ( $\$ 11$ BDSG) organisieren und unter Umständen die Informationspflichten bei Datenschutzverstößen ( $\mathbb{S} 42$ a BDSG) erfüllen. Alles unter Berücksichtigung der Grundsätze der Datenvermeidung, Datensparsamkeit ( $\int 3 a$ BDSG), Zulässigkeit, Zweckbindung und Erforderlichkeit der Datenerhebung, Datenverarbeitung und Datennutzung ( $\$ 28$ BDSG).

Als übergeordnete Stelle fungiert die Aufsichtsbehörde für den nicht öffentlichen Bereich in dem jeweiligen Bundesland. Diese ist sowohl Kontrollinstanz, außerbetriebliche Beschwerdestelle in Fällen von Datenschutzverstößen, aber auch beratende Stelle in Detailfragen zum Datenschutz ( $\int 38$ BDSG).

\section{Was tun?}

Die Fülle der Aufgaben zeigen die erforderlichen Kenntnisse eines Datenschutzbeauftragten. Mit der Erfüllungsvoraussetzung der Zuverlässigkeit und der Fachkunde ( $\$$ 4f Abs. 2 BDSG) wird meist ein interner Mitarbeiter zum betrieblichen Datenschutzbeauftragten bestellt, natürlich mit einem erforderlichen zeitlichen Budget zur Aufgabenerledigung, denn eine Pro-forma-Bestellung ohne Tätigkeitsnachweis wird von Aufsichtsbehörden nicht akzeptiert.

Es gibt aber auch Varianten: Hilfreich kann eine Begleitung durch einen externen Berater zum Datenschutz sein - sowohl beratend als auch im Sinne eines Projektmanagements bis als externer Datenschutzbeauftragter. Bei der Auswahl geeigneter Partner sollte hier neben der Fachkunde als Datenschutzbeauftragter auch die Kenntnis und Fachlichkeiten der Branche eine Rolle spielen.

\section{Ausblick}

Der vorstehende Beitrag beschreibt die Organisation des Datenschutzes in sozialen Einrichtungen zusammenfassend und lässt vermutlich manche Detailaufgaben unbeantwortet. So ergeben sich derzeit viele Themen aus der Praxis mit Handlungsbedarf, wie beispielsweise die Verwendung von Social Media, die Themen der Verpflichtung zum Datenschutz für Mitarbeitende, die Einwilligung der betroffenen Mitarbeitenden und Nutzer, die Entbindung von der Schweigepflicht, E-Mail-Verschlüsselung, Archivierungsund Löschpflichten und vieles mehr.

Datenschutz in sozialen Einrichtungen ist eine Verpflichtung gegenüber dem Klienten und deren anvertrauten
Daten. Datenschutz geht mit der Sozialen Arbeit konform, muss aber organisatorisch geregelt und gelebt werden. Dann kann Datenschutz zu einem Qualitätsmerkmal der Einrichtung werden, nicht nur als Argument zur Rechtssicherheit im Unternehmen.

Das Bundesdatenschutzgesetz steht im Internet im Wortlaut frei zugänglich zur Verfügung: www.gesetze-im-internet.de/ bdsg_1990.

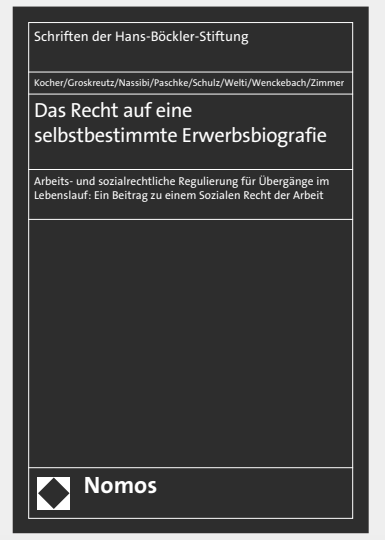

\section{Das Recht auf eine selbstbestimmte Erwerbsbiografie}

Arbeits- und sozialrechtliche Regulierung für Übergänge im Lebenslauf: Ein Beitrag zu einem Sozialen Recht der Arbeit

Von Eva Kocher, Henning Groskreutz, Ghazaleh Nassibi, Christian Paschke, Susanne Schulz, Felix Welti, Johanna Wenckebach und Barbara Zimmer

2013, 387 S., brosch., 79,- $€$ ISBN 978-3-8487-0611-2

(Schriften der Hans-BöcklerStiftung, Bd. 76)

www.nomos-shop.de/21163

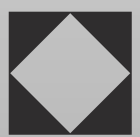
Nomos 